Relations industrielles

Industrial Relations

\title{
Wage Determination, An Analysis of Wage Criteria, by Jules Backman, D. Van Nostrand Company, Inc., N.J., 1959, 316 pp.
}

\section{Jacques St-Laurent}

Volume 16, numéro 1, janvier 1961

URI : https://id.erudit.org/iderudit/1021899ar

DOI : https://doi.org/10.7202/1021899ar

Aller au sommaire du numéro

Éditeur(s)

Département des relations industrielles de l’Université Laval

ISSN

0034-379X (imprimé)

1703-8138 (numérique)

Découvrir la revue

Citer ce compte rendu

St-Laurent, J. (1961). Compte rendu de [Wage Determination, An Analysis of Wage Criteria, by Jules Backman, D. Van Nostrand Company, Inc., N.J., 1959, 316 pp.] Relations industrielles / Industrial Relations, 16(1), 127-127.

https://doi.org/10.7202/1021899ar

Tous droits réservés @ C Département des relations industrielles de l’Université Laval, 1961
Ce document est protégé par la loi sur le droit d'auteur. L'utilisation des services d'Érudit (y compris la reproduction) est assujettie à sa politique d'utilisation que vous pouvez consulter en ligne.

https://apropos.erudit.org/fr/usagers/politique-dutilisation/ 
L'auteur continue ainsi pour les autres critères énumérés plus haut. Dans sa conclusion, il souligne que les gages sont beaucoup plus qu'un taux horaire, et qu'un groupe de facteurs complexes détermine l'employé à accomplir son travail. Ses gages sont composés de bénéfices tangibles et intangibles et ses réactions, vis-à-vis les changements dans ces bénéfices, sont imprévisibles.

JeAn-Paul DEschênes

Wage Determination, An Analysis of Wage Criteria, by Jules Backman, D. Van Nostrand Company, Inc., N.J., 1959, 316 pp.

Voilà un livre qui plaira à ceux qui sont intéressés à la détermination des taux de rémunération, sans être particulièrement intéressés à la théorie des salaires, et à ceux surtout qui ont l'occasion de participer à l'élaboration de ces taux au cours de négociations ou d'arbitrage. Le livre, en effet, ne peut rebuter ceux dont l'esprit répugne à l'analyse de choses abstraites, puisqu'on n'y traite aucunement de la théorie des salaires. Celle-là, dit l'auteur, ne sert à rien dans les négociations collectives. Ce dernier se propose donc de ne passer en revue que les principaux critères utilisés par les parties en présence pour accepter ou refuser la prétention de l'autre: Comparaison des salaires, budgets familiaux, coût de la vie, productivité, capacité de payer et contexte économique général. L'intérêt de cette revue est triple: on y trouve pour chacun des critères les différentes façons dont on peut les utiliser, les limites à chacune de ces façons et une référence à leurs sources statistiques.

Les références aux sources statistiques sont, pour la plupart, américaines. Pour cette raison, elles ne sont pas d'une très grande utilité pour les négociateurs canadiens. L'auteur cependant, à diverses occasions, complète son exposé par des données générales sur la situation canadienne.

Le livre dans l'ensemble apporte une contribution valable aux éclaircissements qu'il est nécessaire d'apporter afin que soient utilisés avec plus de correction les critères habituels de détermination des salaires. Pour le lecteur non averti, le livre comporte cependant certaines trappes.
Tout d'abord, l'image des restrictions à l'utilisation de certains critères ou celle de leurs implications n'est pas toujours complète. Je n'en veux citer qu'un exemple. A l'occasion de la discussion sur le critère du coût de la vie, nulle mention n'est faite du délai habituellement courant entre l'augmentation du coût de la vie et l'augmentation des salaires. Ce délai a pour conséquence de ne jamais permettre au travailleur la possibilité de maintenir son standard de vie.

Ensuite, l'analyse est parfois fautive et seul un esprit averti peut en déceler les faiblesses. C'est ainsi que l'affirmation suivante (p. 238) malgré sa sûreté apparente n'a rien de très sûr: $\ll . .$. the increase in the total employment costs in the steel industry between 1945 et 1955 was greater than total profits before taxes in 1955. In other words, if steel prices had not been increased, the industry would have operated at a substantial loss in 1955 s. En effet le maintien des profits - malgré l'augmentation du coût total de l'emploi - peut se réaliser si l'augmentation des quantités produites est plus que proportionnelle à l'augmentation des coûts de l'emploi ou si des changements dans d'autres facteurs de production ont conduit à des économies. Des faits ci-haut mentionnés, il ne s'ensuit pas nécessairement qu'il y a eu augmentation des prix.

Enfin, il nous a semblé que l'auteur. malgré un effort réel et apparent pour présenter le tout de façon objective, n'y réussit pas toujours. On peut déceler dans son texte des sympathies pour l'une des deux parties en cause lors de la détermination des taux de rémunération. Elle se traduit dans le fait que ce qui peut $y$ avoir d'incomplet ou d'incorrect dans son étude est habituellement au désavantage de celui qui offre son travail.

Malgré ces restrictions - d'une certaine importance - il reste quand même que l'auteur apporte clairement une contribution utile à ceux dont c'est le devoir d'éprouver les limites et implications des critères de détermination du salaire.

Jacques St-Laufent

« Automation and the Worker: a Study of Social Change in Power Plants s, by Floyd C. Mann and L. Richard Hoffman, Henry Holt and Co., New York, 1960,272 pp. plus XIV, $\$ 4.50$ 